\title{
The periosteum: what is it, where is it, and what mimics it in its absence?
}

\author{
Jerry R. Dwek
}

Published online: 5 January 2010

(C) The Author(s) 2009. This article is published with open access at Springerlink.com

Nearly every bone in the body is invested in periosteum. The periosteum is in some ways poorly understood and has been a subject of controversy and debate. This tissue has a major role in bone growth and bone repair and has an impact on the blood supply of bone as well as skeletal muscle. Despite the importance of the periosteum is has received little attention in the literature in recent years.

\section{Histology of the periosteum}

Periosteum can be thought of as consisting of two distinct layers, an outer fibrous layer and an inner layer that has significant osteoblastic potential. In 1739, Duhamel noted that silver wires embedded under the periosteum became covered by osseous matrix. Drawing a parallel with trees, whose cambium is responsible for appositional growth and the distinctive ring pattern seen in cut logs, he termed the inner layer of the periosteum, the cambium [1]. Later, in 1867 , Ollier, by showing that the cambium is capable of producing bone when excised as a free periosteal graft, confirmed that the cambium layer is the essential periosteal component responsible for bone growth [2].

\section{Outer layer}

The outer fibrous layer can be subdivided into two parts. The superficial portion is generally inelastic being relatively cell poor with a predominant collagenous matrix and few

\section{J. R. Dwek ( $\square)$}

Rady Childrens' Hospital and Health Center,

University of California at San Diego,

San Diego, CA, USA

e-mail: jdwek@yahoo.com elastic fibers. Rather than the large collagen bundles found in the skin, collagen fibers in the superficial portion consist mainly of small compact bundles with interspersed elongated fibroblasts [3]. It is the most highly vascularized substratum of the periosteum and is a significant contributor to the blood supply of bone and even skeletal muscle. It also includes a rich neural network. Some of these nerve fibers travel with blood vessels through the bone cortex although the majority terminate at the deeper substratum of the fibrous outer layer. The deep portion of the outer layer has been described as the fibroelastic layer since it contains many elastic fibers and so has significant elasticity. It is also highly collagenous but is cell poor and not highly vascularized. Periosteal tendon attachments usually terminate in this fibroelastic substratum [4].

Inner cambium layer

The cambium layer is highly cellular and is composed of mesenchymal progenitor cells, differentiated osteogenic progenitor cells, osteoblasts and fibroblasts in a sparse collagenous matrix. The osteoblasts lie in contact with the cortical surface. Small compact cells resembling fibroblasts are present about the inner osteoblastic cells with a more peripheral rich vascular and neural sympathetic network [3]. Due to its vascular network many endothelial pericytes are present. These have been shown to have osteoblastic potential and may serve as an ancillary source of progenitor cells [5, 6].

The cambium is at its thickest in the fetus and becomes progressively thinner with age. In the adult it becomes so thin that it cannot be distinguished from the overlying fibrous layer [4]. Vessel density and the number of periosteal fibroblasts also decrease with age so that in the adult, periosteum is present only as a very thin tissue enveloping the bony structures [5]. With this atrophy comes 
a decrease in osteoblastic potential. The cambium is exceedingly important for bone growth in the fetus but loses much of its osteoblastic potential during childhood. It can be stimulated and revived after a fracture albeit with a decreased rate of bone formation. In the adult, osteoblasts in the deep layer have a drawn out elongated configuration essentially indistinguishable from the adjacent fibroblasts.

Interestingly, osteoblastic potential differs not just with age but also by location. Work with periosteal free grafts has shown that calvarial periosteum has less osteogenic potential than that of the tibia $[1,7]$.

\section{Developmental anatomy of the periosteum}

Anatomically, periosteum covers the majority of the bony structures with the exception of their intra-articular surfaces and sesamoid bones. To understand this it is helpful to review the embryology and formation of the long bones and the development of joints.

Bone formation occurs by two processes, membranous or endochondral ossification. In some bones both processes occur. Membranous ossification occurs mostly in the bones of the cranial vault, the mandible, maxilla, and the middle of the clavicle. It refers to the fact that ossification occurs from mesenchyme without the formation of an intervening cartilage model. During ossification the periphery of the mesenchymal model condenses to form the double-layered periosteum. The margins of the periosteum can be clearly elucidated by the examination of the margins of cephalohematoma in infants. These subperiosteal hemorrhages are delimited by the sutures to whose margins the periosteum is attached.

Most of the long bones undergo both endochondral and membranous ossification. Initially during the process of mesenchymal chondrification, the mesenchyme condenses to a cartilaginous model of the long bone. In the center of the cartilaginous model, chondrocytes swell, die, and then calcify with formation of a physis at each end of the ossific nucleus, also termed the primary center of ossification. The physis contains a zonal layered arrangement in which the process of cell hypertrophy, death, and calcification is played out progressively as the ossification front progresses to the ends of the bone. At the beginning of this process, the perichondrium forms as a cellular condensation along the periphery of the cartilaginous model. With vascular invasion, chondrocytes in this layer differentiate into osteoblasts so that at that point a periosteum can be said to exist. By a process identical to membranous ossification the periosteum begins producing a thin peripheral layer of bone termed the bone bark. Along with the longitudinal growth of bone from within occurring by means of endochondral ossification the bone bark advances along the periphery of the bone by membranous ossification, thereby enclosing the nascent cartilaginous model in a bony shell and contributing to appositional growth $[8,9]$. The bone bark is also termed the periosteal or perichondrial collar and is the same structure as the ring of Lacroix, which encircles the physis.

At the ends of the cartilaginous model, cavitation to form the joint cavity is well on its way, having begun during the process of mesenchymal chondrification. As cavitation occurs at the ends of the mesenchymal/cartilaginous model the articular surfaces at the ends of bones are left without a periosteum, thereby allowing development of the articular cartilage [10]. As the joint cavitates, the fibrous capsule is formed along the periphery of the mesenchymal/ cartilaginous model just as the periosteum/perichondrium formed more proximally along the same nascent bony model. It is demonstrated from the previous that the perichondrium, later to become periosteum, as well as the fibrous capsule of the joint, have similar developmental pathways. This is likened to a sleeve around the forming mesenchymal/ cartilaginous/bony model, emphasizing from an embryological vantage point that periosteum, perichondrium, and fibrous capsule should be continuous, as indeed they are.

In the child, the periosteum extends along the primary center of ossification of a bone to the level of the physis at which point it is tightly attached. More proximally along a long bone its attachment to the bony cortex via Sharpey's fibers is more loose and flimsy. This fact is responsible for the production of Salter 2 fractures in which a fracture running though the physis is unable to violate the tight periosteal attachment to the physis and deviates into the metaphysis creating the so-called "Thurston-Holland" metaphyseal fragment. In child abuse, the fracture running along the zone of provisional calcification also frequently deviates into the metaphysis proper and again a metaphyseal fragment is formed. Because of the loose periosteal attachment proximally, considerable subperiosteal hemorrhage may occur.

Surrounding the physis is the groove of Ranvier and the bone bark. The groove of Ranvier contains a zonal arrangement of cells encircling the end of the physis. It contributes both osteoblasts to the bone bark, which continues to cause appositional growth and chondrocytes to the epiphysis, thereby enlarging that structure as well.

The specific point of tight periosteal attachment is the cartilaginous epiphysis just beyond the groove of Ranvier with the fibrous layer lending structural integrity to the groove as it covers it. The fibrous layer continues onward onto the child's cartilaginous epiphysis as the perichondrium. The perichondrium is not easily separable into an outer and inner layer, but has significant chondrogenic potential [11]. At the level of the joint capsule the perichondrial periosteal continuum is itself continuous with the joint capsule [12]. 
At the end of puberty with physeal closure the perichondrium, now enclosing the completely ossified epiphysis transforms into periosteum. It remains continuous with the fibrous capsule of joints so that after physeal closure only the extra-articular portions of secondary centers of ossification are sheathed in periosteum. An example of this can be seen in the knee where corticoperiosteal free flaps are frequently harvested from the distal femur. The flap is dissected down to the level of the superficial band of the medial collateral ligament on the medial surface of the distal femur. Since the superficial medial collateral ligament inserts in adults distal to the physeal margin periosteum is present at least down to this level of the extra-articular epiphysis [13, 14].

It can be seen therefore that the entire length of long bones are sheathed in periosteum with the exception of the intra-articular portion of the bone. The hip joint would seem to be a curious exception since in the adult, the femoral neck is intra-articular. In that case, what contributes to the bone bark and appositional growth if there should be no periosteum along the femoral neck? Indeed, what is the nature of the periosteal attachment to the physis at the proximal femoral physis? The answer is supplied by work performed by Johnson et al. in 1989. In their article, which details anatomical and MR imaging correlation of the infant hip joint, the periosteum is described as continuous with the fibrous capsule of the hip joint, but both are reflected back along the femoral neck. They fuse partially and retain a tight attachment at the level of the physis so that periosteum still lies along the femoral neck cloaked by the reflected portion of the fibrous capsule. The capsular attachment migrates inferiorly as the hip develops [15]. This may also serve to explain confusing reports that have surfaced describing mineralizing periosteal tissue, calcifying fibrocartilage, and expression of alkaline phosphatase along the femoral neck in the hip joints of adults [16-18].

The sesamoid bones are a special case. Sesamoid bones such as the patella form as a cartilaginous condensation along one surface of a tendon, in the case of the patella, the prepatellar quadriceps continuum [19]. The continuum encompasses the patellar extension of fibers largely derived from the rectus femoris, which forms the patellar tendon below. Andersen, writing in 1961, showed histologically that the developing patella in human fetuses is not surrounded by a perichondrium [20]. Bland and Ashhurst working with rabbit fetuses verified that at no point during formation does a periosteum or perichondrium exist along the anterior surface of the patella. Instead the attachment of the tendon to the patella is fibrocartilaginous [19]. The thin sclerotic line seen along the dorsal border of the patella represents the calcified layer of fibrocartilage similar to the tidemark and deep calcified zone of articular cartilage. Recently, Wangwinyuvirat et al. confirmed this finding in an analysis of the histology of the prepatellar quadriceps attachment to the patella [21]. The posterior surface of the patella is covered by articular cartilage so that it too is devoid of periosteum. References in the literature to "periosteal sleeve" avulsions of the inferior patellar pole are mistaken. It is in fact a sleeve of epiphyseal cartilage along with the bone forming physeal cartilage and a zone of provisional calcification that is responsible for the distinctive picture of a thin curvilinear ossific fragment displaced from the inferior patellar pole [22].

\section{Periosteal substitutes}

The periosteum and its precursor, perichondrium, have two major functions aside from lending some structural integrity to the skeleton. Embryologically, both periosteum and perichondrium have major impacts on skeletal development with strong contributions to the appositional growth of bones. In addition, periosteum provides essential cellular and biological components necessary for fracture healing and bone repair.

In bones where neither periosteum nor perichondrium are present, what functions in its place? How does appositional growth occur without the osteogenic and chondrogenic potential of the perichondrium and periosteum? For that matter, in sesamoid bones or the intra-articular portions of the frame not covered by periosteum or articular cartilage, what causes regeneration of bone after traumatic loss? In order to understand the answers to these questions one must consider the contribution of the spherical epiphyseal physis to appositional growth and examine closely the various primary mechanisms of bone repair.

\section{Appositional growth}

Secondary centers of ossification are frequently largely intra-articular structures and therefore, like sesamoid bones, are largely devoid of periosteum. Such is also the case with the carpal bones, which only have small patches of periosteum on the non-articular surfaces. All these bones have a spherical growth plate that rings the entire ossific center and it is this structure that is responsible for an increase in diameter of these largely round or oblong ossific structures. When examining these bones by MRI in the growing child the spherical growth plate is apparent as a bright structure lying along the outer surface of the ossific nucleus using fluid-sensitive sequences. These bones are complete centers of endochondral ossification with no contribution of membranous ossification from periosteum. It is the spherical growth plate that is responsible for appositional growth in these bones, which lack a periosteal covering. 
Fracture healing

While it is true that periosteum and periosteally derived cells are major contributors to fracture healing, it is by no means the only mechanism available to the osseous structures for its repair. The several processes of bone repair have been recently well summarized by Shapiro [23]. Endochondral bone repair is the primary form of bone repair where periosteum is the major player. After a fracture, a hematoma forms, which is stabilized by the surrounding soft tissues and a short time later by a reconstituted (if it is indeed torn) fibrous layer of the periosteum. Cells from the inner cambium layer proliferate and differentiate. At the periphery of the fracture, where good vascularity is present, the inner periosteal layer lays down a collar of bone by the process of membranous ossification. Nearer to the fracture site, the cambium produces a mass of cartilage about the fracture site. Then, by a process of endochondral ossification that recapitulates skeletogenesis in the fetus the cartilage mass is ossified [24]. This type of repair dominates in situations where there is either macromotion or micromotion of the fractured parts in periosteally clad bones. It is the type of repair that is radiographically evident as a periosteal reaction, which includes an orderly progression from subperiosteal new bone formation, soft callus, which is radiolucent within, and hard callus with loss of definition of the fracture planes. Periosteal reaction can occur anywhere periosteum is present, although it is unclear why in the adult, periosteal reaction usually does not extend to the level of the metaphysis and extra-articular epiphysis as can be seen in many healing fractures, and other situations where periosteal reaction is thought to be present such as hypertrophic osteopathy. Periosteal reaction does not occur in bones that lack a periosteum.

In situations where little or no motion is present and the fractured ends are closely apposed, two types of repair mechanisms occur. When no gap at all is present between the fractured cortical ends primary bone repair occurs by dint of osteoprogenitor cells derived directly from the Haversian systems within the cortex. This repair mechanism is also termed contact repair and produces lamellar bone already oriented correctly along the long axis of the bone. Gap repair or direct transformational bone repair comes into play when a small gap is present between the fractured ends. In this form, lamellar bone is formed initially at right angles to the bone and is then remodeled to the correct orientation. In wider gaps, woven bone is first produced and transformed to lamellar bone. In neither case is there a cartilage intermediary model.

Lastly, callotasis is the process utilized in leg lengthening procedures. This type of distraction osteogenesis utilizes primary periosteal contribution, but as opposed to endochondral bone repair, no cartilaginous fracture mass is formed. Instead woven bone is produced from a mixture of mesenchymal osteoblastic cells and remodeled to standard lamellar bone.
While it is true that healing is delayed when fractures are experimentally produced after stripping of the periosteum, healing is also delayed when the marrow cavity is reamed. However, neither the former nor the latter experimentally produced injury alone will obviate healing [24-27]. Clearly, fracture healing is a complex multifactorial process.

While in many cases of fracture healing it is the cambium layer that supplies many of the chondrogenic and osteoblastic cells, there are many other sources. The endosteum can be conceived as a connective tissue reticulum and consists of cells with osteoblastic potential lining all of the interior surfaces of bones including the inner surface of the cortex, the trabeculae, and the haversian systems [4]. Not only is this a potent source of osteogenic cells, but it may in certain cases be a more potent source leading to faster fracture healing, as is the case in metaphyseal or epiphyseal fractures involving the spongiosa. In that richly vascular environment, with a vast expanse of surface area harboring many osteogenic cells, fracture healing is faster than when fracture occurs in compact bone [23]. Besides the endosteum a partial list of other osteogenic cell sources include undifferentiated marrow cells that have access to the blood circulation [28], pericytes [6], and undifferentiated cells in adjacent soft tissues including muscles [29], fascia, and blood vessels [30]. All these play a role, along with a panoply of hormonal growth factors in bony repair mechanisms.

\section{Conclusion}

The periosteum is a complex structure composed of an outer fibrous layer that lends structural integrity and an inner cambium layer that possesses osteogenic potential. During growth and development it contributes to bone elongation and modeling, and when the bone is injured, participates in its recovery. It is not, however, present in all bones - sesamoid bones and the intra-articular ends of bone are notable exceptions. In these areas, other growth and repair mechanisms are substitutes for the absent periosteum.

Open Access This article is distributed under the terms of the Creative Commons Attribution Noncommercial License which permits any noncommercial use, distribution, and reproduction in any medium, provided the original author(s) and source are credited.

\section{References}

1. Bilkay U, Tokat C, Helvaci E, Ozek C, Zekioglu O, Onat T, et al. Osteogenic capacities of tibial and cranial periosteum: a biochemical and histologic study. J Craniofac Surg. 2008;19(2):453-8. 
2. Ito Y, Fitzsimmons JS, Sanyal A, Mello MA, Mukherjee N, O'Driscoll SW. Localization of chondrocyte precursors in periosteum. Osteoarthritis Cartilage. 2001;9(3):215-23.

3. Squier CA, Ghoneim S, Kremenak CR. Ultrastructure of the periosteum from membrane bone. J Anat. 1990;171:233-9.

4. Jaffe H. Metabolic, degenerative and inflammatory diseases of bones and joints. Philadelphia: Lea \& Febiger; 1972.

5. Allen MR, Hock JM, Burr DB. Periosteum: biology, regulation, and response to osteoporosis therapies. Bone. 2004;35(5):1003-12.

6. Diaz-Flores L, Gutierrez R, Lopez-Alonso A, Gonzalez R, Varela H. Pericytes as a supplementary source of osteoblasts in periosteal osteogenesis. Clin Orthop Relat Res. 1992;275:280-6.

7. Uddstromer L. The osteogenic capacity of tubular and membranous bone periosteum. A qualitative and quantitative experimental study in growing rabbits. Scand J Plast Reconstr Surg. 1978;12 (3):195-205.

8. Resnick D, Manolagas S, Fallon M. Histogenesis, anatomy and physiology of bone. In: Resnick D, editor. Diagnosis of bone and joint disorders. 4th ed. Philadelphia: W.B. Saunders Company; 2002. p. 648-54.

9. Williams P, Warwick R. Gray's Anatomy, 36th ed. Philadelphia: W.B. Saunders Company; 1980. p. 260-4.

10. Williams N. Textbook of rheumatology. Philadelphia: W.B. Saunders Company; 1981.

11. Shapiro F, Holtrop ME, Glimcher MJ. Organization and cellular biology of the perichondrial ossification groove of ranvier: a morphological study in rabbits. J Bone Joint Surg Am. 1977;59 (6): $703-23$

12. Davies DV. The anatomy and physiology of joints. Physiotherapy. 1963;49:3-7.

13. Fuchs RK, Allen MR, Ruppel ME, Diab T, Phipps RJ, Miller LM, et al. In situ examination of the time-course for secondary mineralization of Haversian bone using synchrotron Fourier transform infrared microspectroscopy. Matrix Biol. 2008;27(1):34-41.

14. Choudry UH, Bakri K, Moran SL, Karacor Z, Shin AY. The vascularized medial femoral condyle periosteal bone flap for the treatment of recalcitrant bony nonunions. Ann Plast Surg. 2008;60 (2): $174-80$

15. Johnson ND, Wood BP, Noh KS, Jackman KV, Westesson PL, Katzberg RW. MR imaging anatomy of the infant hip. AJR Am J Roentgenol. 1989;153(1):127-33.

16. Allen MR, Burr DB. Human femoral neck has less cellular periosteum, and more mineralized periosteum, than femoral diaphyseal bone. Bone. 2005;36(2):311-6.
17. Power J, Loveridge N, Rushton N, Parker M, Reeve J. Evidence for bone formation on the external "periosteal" surface of the femoral neck: a comparison of intracapsular hip fracture cases and controls. Osteoporos Int. 2003;14(2):141-5.

18. Shea JE, Vajda EG, Bloebaum RD. Evidence of a hypermineralised calcified fibrocartilage on the human femoral neck and lesser trochanter. J Anat. 2001;198(Pt 2):153-62.

19. Bland YS, Ashhurst DE. Fetal and postnatal development of the patella, patellar tendon and suprapatella in the rabbit; changes in the distribution of the fibrillar collagens. J Anat. 1997;190(Pt 3):327-42.

20. Andersen H. Histochemical studies on the histogenesis of the knee joint and superior tibio-fibular joint in human foetuses. Acta Anat (Basel). 1961;46:279-303.

21. Wangwinyuvirat M, Dirim B, Pastore D, Pretterklieber M, Frank A, Haghighi P, et al. Prepatellar quadriceps continuation: MRI of cadavers with gross anatomic and histologic correlation. AJR Am J Roentgenol. 2009;192(3):W111-6.

22. Bates DG, Hresko MT, Jaramillo D. Patellar sleeve fracture: demonstration with MR imaging. Radiology. 1994;193(3):825-7.

23. Shapiro F. Bone development and its relation to fracture repair. The role of mesenchymal osteoblasts and surface osteoblasts. Eur Cell Mater. 2008;15:53-76.

24. Ferguson C, Alpern E, Miclau T, Helms JA. Does adult fracture repair recapitulate embryonic skeletal formation? Mech Dev. 1999;87(1-2):57-66.

25. Oni OO, Gregg PJ. An investigation of the contribution of the extraosseous tissues to the diaphyseal fracture callus using a rabbit tibial fracture model. J Orthop Trauma. 1991;5(4):480-4.

26. Oni OO, Stafford H, Gregg PJ. A study of diaphyseal fracture repair using tissue isolation techniques. Injury. 1992;23(7):467-70.

27. Stafford H, Oni OO, Hay J, Gregg PJ. An investigation of the contribution of the extraosseous tissues to the diaphyseal fracture callus using a rabbit tibial fracture model and in situ immunocytochemical localisation of osteocalcin. J Orthop Trauma. 1992;6 (2):190-4.

28. Friedenstein AY. Induction of bone tissue by transitional epithelium. Clin Orthop Relat Res. 1968;59:21-37.

29. Gerstenfeld LC, Cullinane DM, Barnes GL, Graves DT, Einhorn TA. Fracture healing as a post-natal developmental process: molecular, spatial, and temporal aspects of its regulation. J Cell Biochem. 2003;88(5):873-84.

30. Matsumoto T, Kuroda R, Mifune Y, Kawamoto A, Shoji T, Miwa $\mathrm{M}$, et al. Circulating endothelial/skeletal progenitor cells for bone regeneration and healing. Bone. 2008;43(3):434-9. 